\title{
An experimental reinvestigation of the thermodynamics of the field induced SDW in (TMTSF) ${ }_{2} \mathrm{ClO}_{4}$
}

\author{
U. SCHEVEN, W. KANG and P.M. CHAIKIN \\ Department of Physics, Princeton Universty, Princeton NJ 08544, U.S.A.
}

\begin{abstract}
The unique nature of the spin density wave transitions induced by a moderate magnetic field in the Bechgaard salts (TMTSF) $2 \mathrm{X}$ (where $\mathrm{X}=\mathrm{PF}_{6} \mathrm{ClO}_{4}$, etc.) result from competition between the length scales set by the magnetic field and the SDW wavevector and possibly the lattice periodicity. This complex competition has led to experimental and theoretical suggestions of a phase diagram with some novel features including reentrance, arborescence and multicritical points. We have performed high resolution heat capacity and magnetocaloric measurements to reinvestigate these questions. We find no evidence for arborescence, but clear evidence of low field reentrance and a tetracritical point (as in previous work by Pesty et al.) indicating coexistence of order parameters for several FISDW phases.
\end{abstract}

Within the past decade a spectacular set of phenomena have been found in the quasi-one - quasi-two dimensional organic conductors known as the Bechgaard salts (TMTSF) 2 X especially in the presence of applied magnetic fields. The generic behavior in these materials is well represented by the prototype salt (and first organic superconductor) (TMTSF) $2 \mathrm{PF}_{6}$ (1). At ambient pressure it is a highly anisotropic metal which upon cooling to $12 \mathrm{~K}$ becomes an antiferromagnetic insulator through a spin density wave transition. Above a critical pressure $\left(\mathrm{p}_{\mathrm{c}} \sim 6 \mathrm{kbar}\right.$ for $\left.(\mathrm{TMTSF})_{2} \mathrm{PF}_{6}\right)$ the SDW is suppressed, the metallic state reappears and the samples go superconducting at about $1.3 \mathrm{~K}$. Application of a moderate magnetic field this metallic state introduces new phenomena. Transport measurements indicate a cascade of transitions which reduce the number of carriers in a series of steps. In each new phase the Hall resistance is quantized (2).

Our present understanding of these Field Induced Spin Density Wave (FISDW) transitions is contained in a "Standard Model" (3) which has evolved from several theoretical groups. The highly anisotropic bands lead to a Fermi surface consisting of two warped sheets. At ambient pressure the sheets nest sufficiently well when translated by the SDW wavevector that the SDW gap opens over the entire Fermi surface lowering the electronic energy and stabilizing the SDW distortion. At higher pressure the warping is more important, the nesting is imperfect (leaving pockets of electrons or hole or both), there is no gap at $\varepsilon_{\mathrm{F}}$, and the SDW is suppressed. Since there are no closed orbits, it might also be expected that a magnetic field would have little effect. However, if there is an SDW distortion and a magnetic field, the closed pockets of electrons and holes must quantize into Landau levels. Adjusting the SDW wavevector so that the pockets have exactly the right area for an integer number of Landau levels puts $\varepsilon_{F}$ in the gap 
between them. The electronic system gains energy and the SDW is again stabilized. When the magnetic field changes the SDW wavevector changes to make sure that the quantization condition is met. This is the same as assuring that the SDW wavevector deviates from the value $2 \mathrm{kF}_{\mathrm{F}}$ that it would have in a conventional SDW by an integral number of reciprocal magnetic lengths ( $G=2 \pi / \lambda_{\mathrm{m}}=2 \pi \mathrm{eBb} / \mathrm{hc}$ ): $\mathrm{qSDW}_{\mathrm{SD}}$ $=2 \mathrm{kF}_{\mathrm{F}}+\mathrm{nG}$, where, $\mathrm{B}$ is the applied field and $\mathrm{b}$ is a lattice constant in the second most conducting direction. The SDW wavevector varies smoothly with field until at some point it becomes energetically favorable to jump to the next quantum number, and $n$ goes to $n-1$.

The thermodynamic nature of this beautiful cascade of SDW - QHE transitions was confirmed by magnetization (4) and specific heat measurements on (TMTSF) ${ }_{2} \mathrm{ClO}_{4}$ (5). (In this material $\mathrm{p}_{\mathrm{c}}=0$.) However, after the initial confirmation of the phase diagram predicted by the standard model, the specific heat and magnetocaloric studies showed that the transitions were even more complex than had been thought. In particular, the calorimetry group at Orsay found several novel features including (5): reentrance of the normal state in the low field region (i. e. the phase diagram has regions of negative slope in $\mathrm{dT}_{\mathrm{FISDW}} / \mathrm{dB}$ ), oscillations of the strong coupling parameter with field (i.e. the ratio of the specific heat jump to the density of states which in weak coupling is equal to 1.43 as in the BCS theory, here oscillates with the period of the phase boundary, and has a very enhanced value above 7.5 Tesla), and probably most interestingly they found that the phase boundaries seemed to bifurcate as temperature was lowered giving a "tree-like" or "arborescent" aspect to the phase diagram.

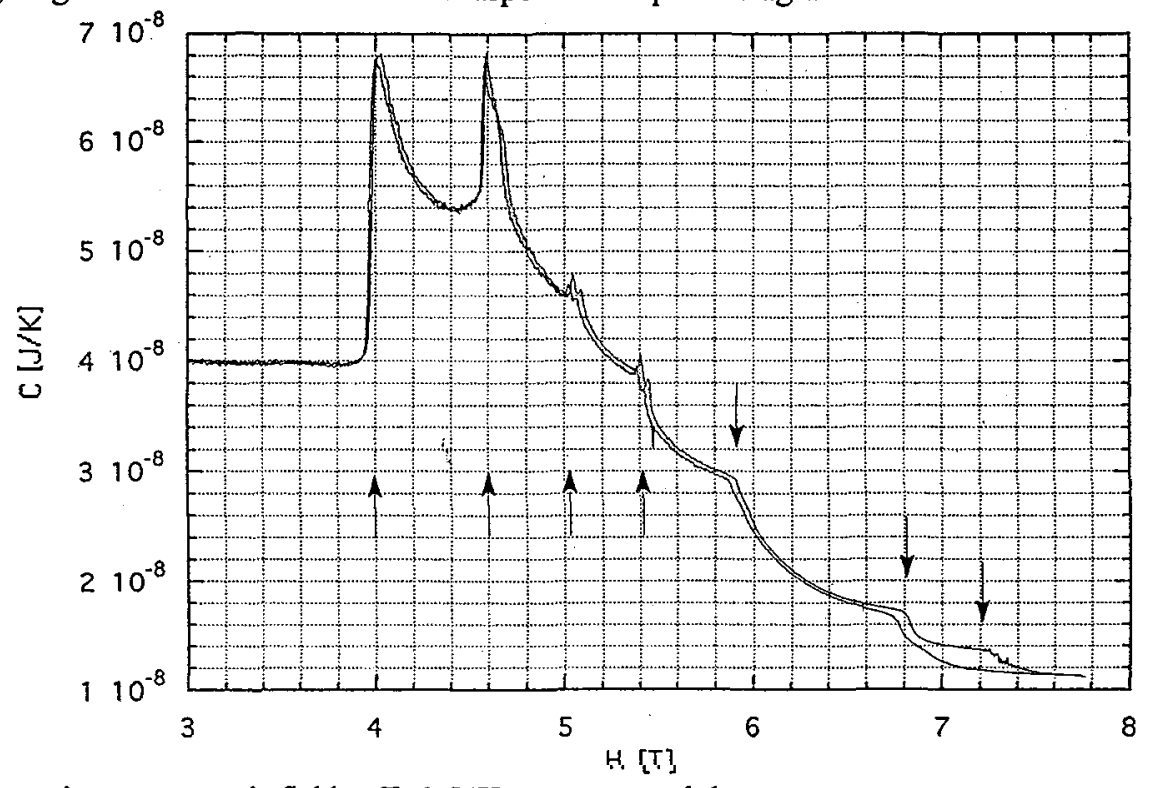

Fig. 1. Heat capacity vs. magnetic field at $\mathrm{T}=0.54 \mathrm{~K}$, upsweep and downsweep.

The arborescence was intriguing theoretically. The Standard model already has a competition of two lengths, $1 / 2 \mathrm{k}_{\mathrm{F}}$ and the magnetic length $2 \pi / \mathrm{G}$, but there is another natural length in the problem, the lattice constant, which should be particularly important in these salts where $2 \pi / 4 \mathrm{kF}$ is a lattice constant. Herritier et al (6) then demonstrated that if the SDW gaps were not small then the nesting condition could be generalized to $\mathrm{mqSDW}_{\mathrm{SD}}=\mathrm{m} 2 \mathrm{k}_{\mathrm{F}}+\mathrm{nG} \rightarrow \mathrm{q}_{\mathrm{SDW}}=2 \mathrm{k}_{\mathrm{F}}+(\mathrm{n} / \mathrm{m}) \mathrm{G}$ and that a complex bifurcating phase diagram would result from the "fractional " quantization of the Landau levels. Later Lebed (7) suggested that the phase diagram might allow the coexistence of order parameters for more that one SDW at a time. Within a simple two order parameter Ginzburg-Landau model he predicted that the standard model's first order transitions between FISDW's with quantum numbers $n$ and $n-1$ would give way to tetracritical points with second order transitions between phases such that $\Delta_{n} \rightarrow \Delta_{n}+\Delta_{n-1} \rightarrow \Delta_{n-1}$. The existence of these tetracritical points has since been confirmed by reanalysis of the thermodynamic data. Later theories 
also found evidence for an arborescence arising from the presence of many subsidiary gaps and order parameters (7).

The present calorimetric studies were performed in the vacuum space below the $\mathrm{He}^{3}$ pot of a $\mathrm{He}^{3}$ refrigerator using AC calorimetry. A $4.7 \mathrm{mg}$ single crystal of (TMTSF) ${ }_{2} \mathrm{ClO}_{4}$ was sandwiched between a RuO metal film thermometer and a metal film heater. The thermometer and heater had been ground down from the original microchip to $\sim 0.5 \mathrm{mg}$ each. Contact between sample and thermometer and heater was made with apiezon $\mathrm{N}$ grease. The four electrical contacts to the thermometer and two to the heater were soldered to the chips. The leads were low thermal conductivity constantan. The heater current was supplied at $5 \mathrm{kHz}$ amplitude modulated at $0.5<\mathrm{f}<2 \mathrm{~Hz}$ hence producing a sinusoidal Joule heating at twice the drive frequency $(1-4 \mathrm{~Hz})$. The thermometer resistance was measured with a Lockin amplifier with probe current at $1 \mathrm{kHz}$. The temperature variation at the heater frequency (essentially inversely proportional to the specific heat) was obtained by measuring the output of the $1 \mathrm{kHz}$ thermometer lockin with another lockin referenced to heater frequency $(1-4 \mathrm{~Hz}$.). For measurements of the the magnetocaloric effect the unmodulated temperature was recorded directly from the $1 \mathrm{kHz}$ thermometer lockin. For simultaneous specific heat and magnetocaloric measurements, both the demodulated $(1-4 \mathrm{~Hz})$ $\mathrm{AC}$ signal and the direct signal (time averaged for several periods of AC modulation) from the thermometer lockin were recorded.

In figure 1 we show the specific heat as a function of magnetic field at $0.54 \mathrm{~K}$. Here we see that the specific heat is essentially independent of field below about 4 Tesla. The onset of the cascade of FISDW's is signaled by the sharp jump in specific heat at 4 Tesla, and this is followed by successively smaller jumps on entering higher field SDW phases. In addition the background specific heat is strongly reduced indicating the reduction of degrees of freedom, entropy and electronic density of states as one goes deeper into the FISDW phases. The data represent superposed sweeps up and down in magnetic field. The only place where there is substantial hysterisis is in the range between 6.8 and 7.4 Tesla corresponding to the final two transitions in this low field regime. ( The latter two transitions have variously been called the Ribault anomaly and the $\mathrm{N}=11 / 3$ " state. There is a high field reentrance and additional transitions at fields above 27 Tesla. ) As we will see more explicitly in the magnetocaloric data discussed below these transitions are highly first order. The arrows indicate the fields which we associate with phase boundaries separating the different FISDW states.

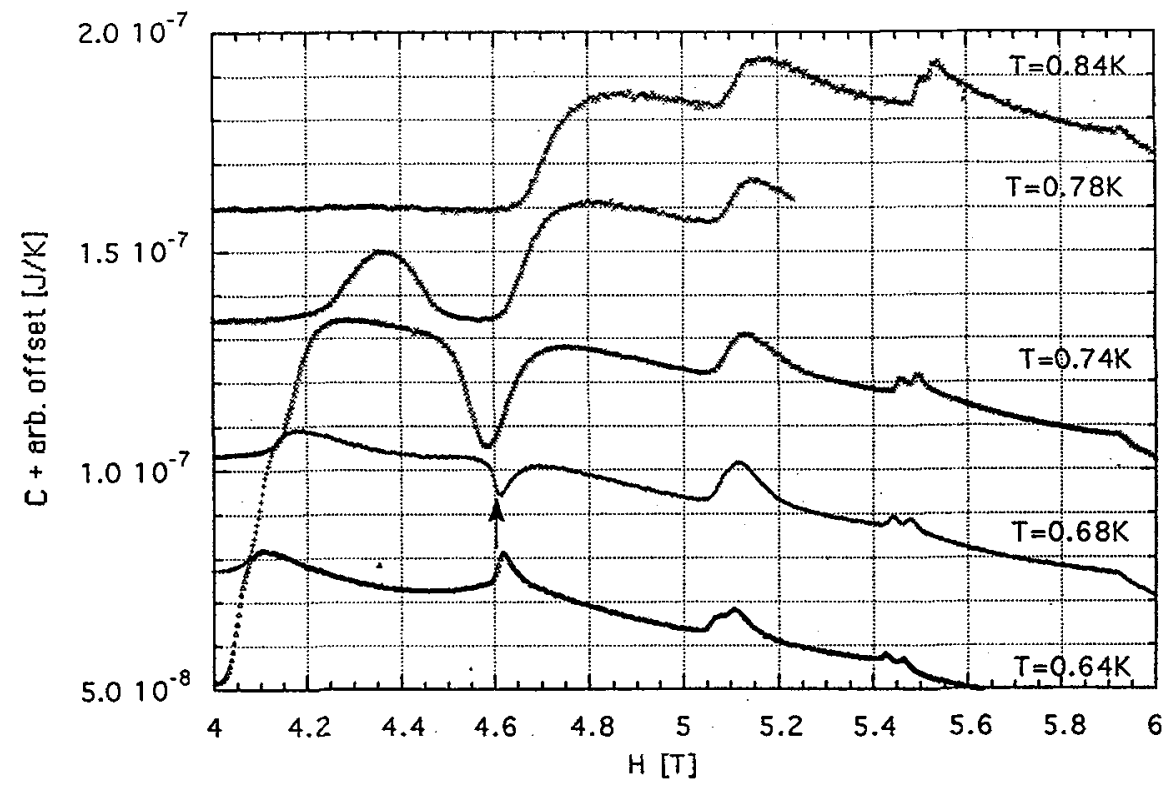

Fig. 2. Heat capacity vs. magnetic field, showing reentrance of the metallic phase. 
Additional $\mathrm{C}_{\mathrm{V}}$. vs $\mathrm{H}$ curves for several temperatures between 0.84 and $0.64 \mathrm{~K}$.in the region of the low field reentrance are shown in figure 2 . Here we see the birth of a new phase and the evolution of the phase boundaries of established phases with decreasing temperature. Note the region between 4 and 4.6 Tesla. At $0.84 \mathrm{~K}$ this is the normal metallic phase. At $0.78 \mathrm{~K}$ we see the $\mathrm{C}_{\mathrm{V}}$ rise associated with a new phase at 4.22 Tesla. However, after reaching a maximum at $4.36 \mathrm{Tesla}$, the $\mathrm{C}_{\mathrm{V}}$ value returns to the normal state value at 4.48 Tesla. The reentrant normal state remains until 4.62 Tesla at which field the next FISDW phase is forming. As temperature is lowered the reentrant normal metal region narrows and the last suggestion of reentrance disappears between 0.68 and $0.64 \mathrm{~K}$.

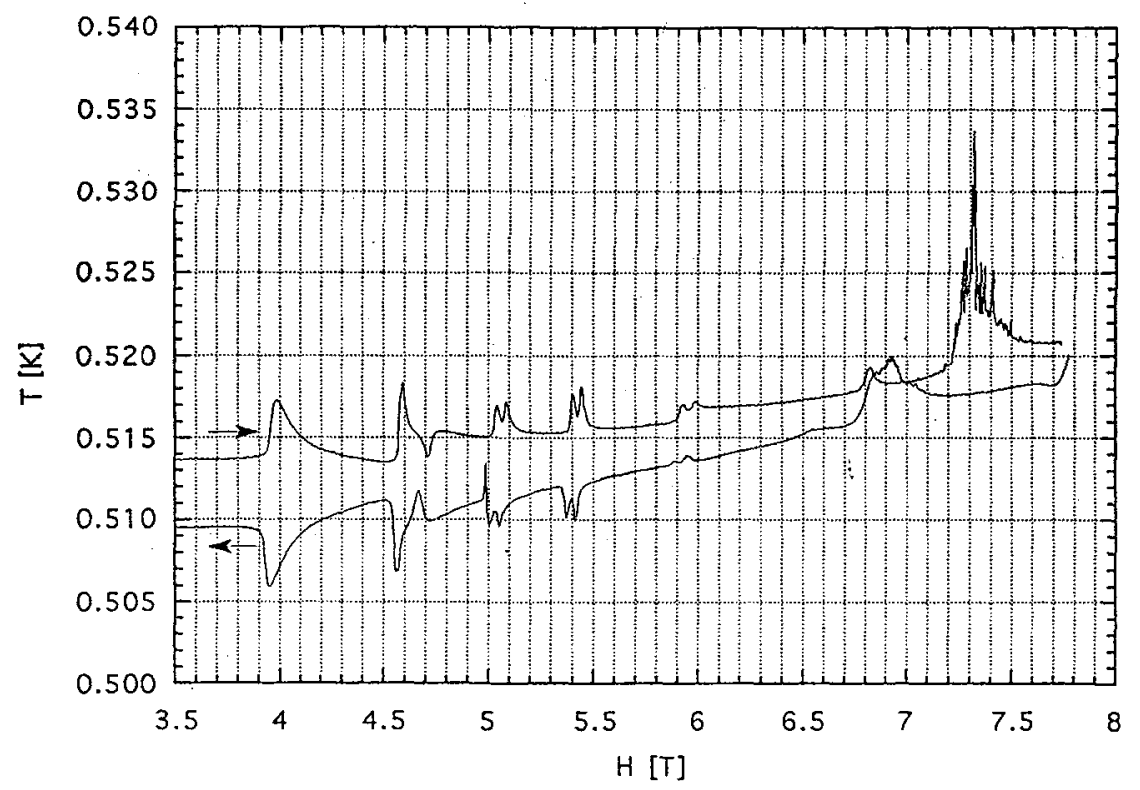

Fig. 3. Temperature vs. magnetic field, magnetocaloric effect, upsweep and downsweep at 36 Gauss/sec.

We now turn our attention to the magnetocaloric data. The basic idea of this experiment is that the sample is weakly connected to a fixed temperature bath while the magnetic field is swept. On entering the lower entropy FISDW state on increasing field, heat is released and the temperature is increased while the heat flows through the thermal link to thermal ground. The temperature increase is proportional to $\partial \mathrm{H} / \partial \mathrm{t}$ times $\partial \mathrm{S} /\left.\partial \mathrm{B}\right|_{\mathrm{T}}$ which by a Maxwell relation is related to $\partial \mathrm{M} /\left.\partial \mathrm{T}\right|_{\mathrm{B}}$. On sweeping the field down the temperature change should be reversed. In figure 3 we show the sample temperature as a function of field for up and down field sweeps. (There is some background dependence due to the magneto resistance of the thermometer.) Between 3 and 5.6 Tesla the up and down sweep are essentially reflections of one another; as expected, and this indicates the reversible nature of the transitions in this region. In figure 4 we show the upsweep magnetocaloric traces for a number of different temperatures. Arrows here indicate the points we have used to construct the phase diagram. Note that many of the phase boundaries are now characterized with two peaks. The entrance to the FISDW state at 4 Tesla is the only single transition. The next transition at 4.6 Tesla is also unusual. There is a sharp temperature increase followed by a sharp temperature decrease. This would indicate that the second of the pair of transitions here is that from leaving an ordered phase. If we follow this pair up in temperature we see that they grow closer together and merge into a single transition at precisely the point where the $\mathrm{C}_{\mathrm{V}}$ data indicated the disappearance of the reentrant transition, or the joining of two phase boundaries from the 
normal metal to the FISDW state. When we put these points on the phase boundary in figure 5 .it is clear that in this region we have a well defined tetracritical point.

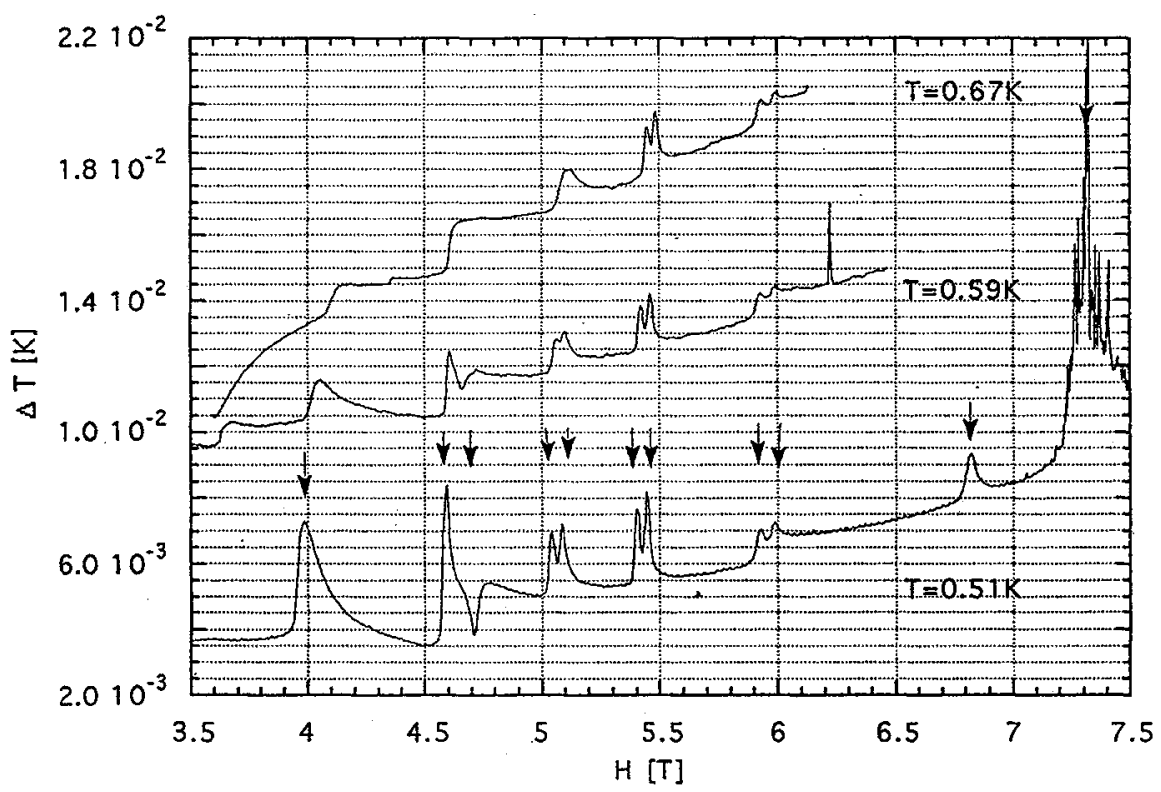

Fig. 4. Temperature evolution vs. magnetic field for 3 different base temperatures. Arrows mark features used to construct the phase diagram in Fig. 5.

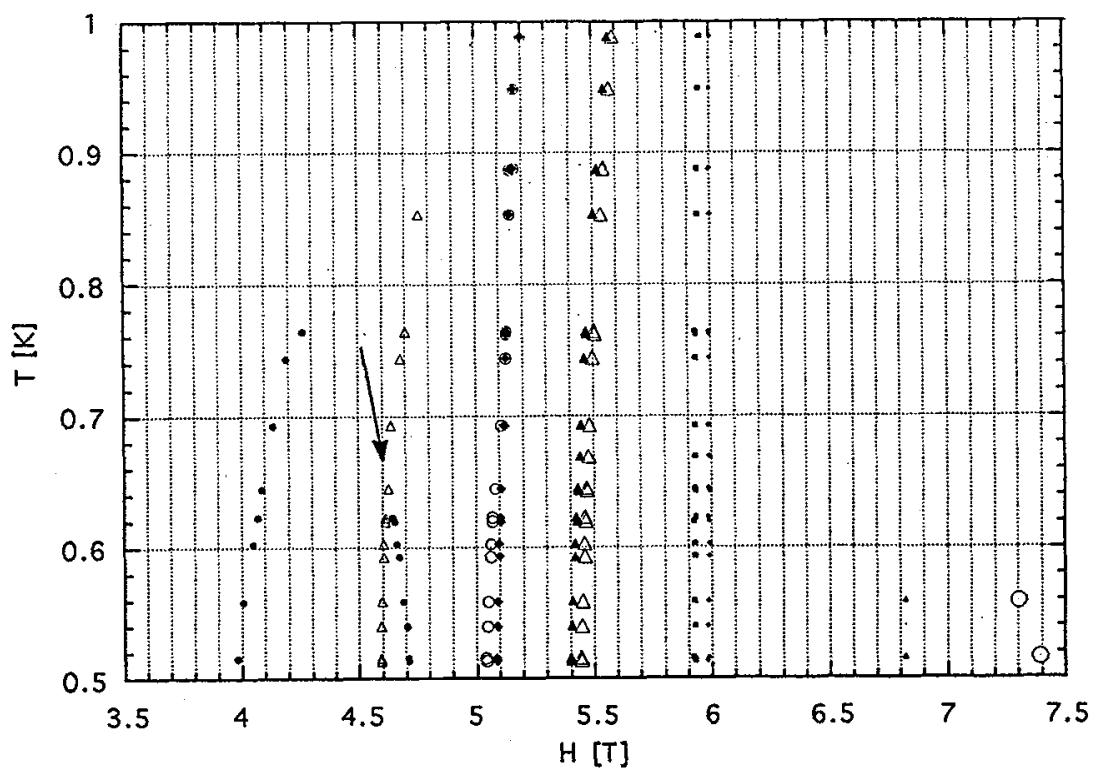

Fig. 5. Partial phase diagram of (TMTSF) ${ }_{2} \mathrm{ClO}_{4}$ based on magnetocaloric data. The arrow marks the point where the last remnants of the metallic reentrance observed in $\mathrm{C}_{\mathrm{V}}$ vanishes 
Returning to the up and down field sweeps in figure 3 we now concentrate on the field region above 5.6 Tesla. Here we have the remarkable result that there are a series of transitions which are not only hysteretic but also are exothermic (heat releasing) for both entering and leaving the more ordered high field state. The upsweep also shows very large temperature spikes corresponding to sudden heat release. These spike are not reproducible in detail, but there are always spikes of the same character when the field is swept.up in this region. We believe that this last (low field) transition is highly first order, but this in itself will not explain the spikes and the irreversibility. We suggest that strong coupling between the FISDW and the phonons (magnetoelastic coupling) may produce sizable internal strains which are relieved by "quakes". The frictional forces associated with these quakes would then account for heat release on either entering or leaving this phase. If this is the case then it should be possible to observe this FISDW by xray scattering from the lattice, an experiment which is presently under way.

Comparing our results with previous studies of the Orsay calorimetry group: we have confirmed their discovery of the cascade of thermodynamic FISDW's, the reentrance of the normal state at $\sim 4.6$ Tesla $\sim .66 \mathrm{~K}$, the existence of a tetracritical point at this point, the doubling of some transition lines and the existence of highly irreversible behavior near 7.5 Tesla at low temperatures. However, our biggest discrepancy with their results is the complete absence of arborescence in our phase diagram. Our samples are larger, and slower cooled (more relaxed), and our signal to noise is about an order of magnitude better than in their experiments. But, between the clear and sharp transitions that we observe there is no sign of anything but smooth behavior. We also find a single reentrance and tetracritical point. The coexistence region of the two phases below the tetracritical point is much narrower in our case than previously described.

In conclusion we find no evidence for arborescence in the otherwise intricate and fascinating phase diagram of (TMTSF) ${ }_{2} \mathrm{ClO}_{4}$. Most of the phase boundaries between reversible subphases show a clear (single) splitting or doubling as temperature is lowered. There is a single tetracritical point (above $0.5 \mathrm{~K}$ ) indicating that some of the FISDW phases can coexist and in the strongest transition there is an unusual heat release on entering or leaving the most ordered phase. We suggest that this latter effect may be the result of coupling of the FISDW to the lattice and the resulting introduction of strain fields at this transition.

\section{References.}

* Present address: AT\&T Bell Labs, Murray Hill, NJ

1. D. Jerome, and H. J. Schultz, Adv. in Phys. 31, (1982) 299.

2. P. M. Chaikin et al., Phys. Rev. Lett., 51,2333 (1983), M. Ribault et al., J. Phys. Lett. 44, (1983) L953

3. L. P. Gor'kov, A. G. Ledel, J. de Phys. Lett. 45, L433 (1984), P. M. Chaikin, Phys. Rev. B31, 4770 (1985), G. Montambaux, M. Heritier, P. Lederer, J. Phys. Lett. 45, L-533 (1984), M. Heritier, G. Montambaux, and P. Lederer, J. Physique Lett. 45, (1984) L-943, K. Yamaji, J. Phys. Soc. Japan 54, (1985) 1034, M. Ya Azbel, Per Bak and P. M. Chaikin, Phys. Lett. Al17, 92 (1986), K. Maki, Phys. Rev. B33, (1986) 4826.

4. M. J. Naughton, Phys. Rev. Lett., 55 (1985) 969.

5. F. Pesty, P. Garoche and M. Heritier, Eds. G. Saito and S. Kagoshima, The Physics and Chemistry of Organic Superconductors, (Springer Proccedings in Physics) 51, (1990) 87, , F. Pesty and P. CGaroche, Fizika, 21 (1989) 40, F. Tsonbnang, F. Pesty, P. Garoche and M. Heritier, Synth. Metals, 41-43 (1991) 1707, F. Pesty, P. Garoche, and K. Bechgaard, Phys. Rev. Lett. 55 (1985) 2495.

6. A. Lebed, JETP Lett. 51 (1990) 663.

7. Y. Hori and K. Machida, J. Phys. Soc. Japan, 61 (1992) 1246 